\title{
Multiplex genome engineering in eukaryotes
}

A goal of synthetic biology is the design and evolution of organisms with new and improved properties, but genomic diversity is difficult to generate efficiently in the laboratory. Multiplex automated genome engineering (MAGE) has been used in prokaryotes to simultaneously target many locations

the precise modification of multiple sites in Saccharomyces cerevisiae 5 cerevisiae without generating DNA double-strand breaks (DSBs).

The majority of techniques for modifying eukaryotic genomes generate DSBs, which limits their ability to simultaneously alter multiple loci in a cell. In Escherichia coli, chromosomes can be modified in the absence of DSBs using synthetic single-stranded DNA (ssDNA) oligodeoxynucleotides (ssODNs) complementary to the lagging strand of the replicating chromosome.

To assess whether ssODNs can be incorporated at replication forks in S. cerevisiae, the authors generated an experimental locus by placing URA 3 (a gene used to label chromosomes) proximal to the origin of replication (Ori) that is adjacent to the gene ADE2 on chromosome 15. Transforming cells harbouring the Ori-URA3-ADE2 locus with ssODNs targeting the predicted lagging strand of both URA 3 and $A D E 2$ revealed that, in the presence of 5-fluoroorotic acid (5FOA), which selects for the absence of Ura3 function, $22 \%$ of colony-forming units contained ssODN-derived ADE2mutations. Thus, in eukaryotes, ssODNs can target DNA replication forks within a contiguous chromosome with greater efficiency than other ssODN incorporation methods.

As previous data showed that homologous recombination of ssODNs results in low allelic replacement frequencies (ARFs) and limits the scope of applications to single locus modifications, the authors created a suite of yeast strains overexpressing or null for factors involved in homologous recombination. Using the Ori-URA3$A D E 2$ locus, they found that the annealing of ssODNs at the replication fork was independent of Rad51, dependent on a ssDNA annealing protein and capable of introducing precise mutations without DSBs. Indeed, under optimal conditions, targeting Ori-URA3-ADE2 with ssODNs could produce an ARF of $47.5 \%$. Silencing DNA repair (by performing experiments in cells null for the DNA mismatch repair protein Msh2) and slowing replication with hydroxyurea were among the factors that enhanced multiplex editing by ssODNs.

Next, the authors carried out three rounds of eMAGE to diversify 50 nucleotide positions within the ADE2 gene and showed that eMAGE creates more genetic diversity with each round. Indeed, $>10^{5}$ unique variants could be generated from a single eMAGE experiment. One round of eMAGE with ssODNs encoding mutations across the genes and regulatory elements in the $\beta$-carotene pathway resulted in many examples of precise multiplex genetic modifications leading to phenotypic variation, including enhanced accumulation of $\beta$-carotene.

It will be interesting to see the impact of eMAGE on genome design and synthesis in yeast and single mammalian cells, and whether eMAGE can be adapted to target multicellular eukaryotes.

Katharine H. Wrighton

ORIGINAL ARTICLE Barbieri, E. M. et al. Precise editing at DNA replication forks enables multiplex genome engineering in eukaryotes. Cell http://dx.doi.org/10.1016/j.cell.2017.10.034 (2017) FURTHER READING Haimovich, A. D., Muir, P. \& Isaacs, F. J. Genomes by design. Nat. Rev. Genet. 16, 501-516 (2015) 